\title{
Technè
}

La science au service de l'histoire de l'art et de la préservation des biens culturels

$40 \mid 2014$

Thérapéia. Polychromie et restauration de la sculpture dans l'Antiquité

\section{Couleur, matériau et lumière : la pensée chromatique dans l'Égypte ancienne}

Colour, material and light : chromatic reasoning in Ancient Egypt

\section{Christophe Barbotin}

\section{OpenEdition}

1 Journals

Édition électronique

URL : https://journals.openedition.org/techne/3166

DOI : $10.4000 /$ techne.3166

ISSN : 2534-5168

\section{Éditeur}

C2RMF

\section{Édition imprimée}

Date de publication : 26 novembre 2014

Pagination : 23-29

ISBN : 978-2-7118-6218-4

ISSN : $1254-7867$

Référence électronique

Christophe Barbotin, "Couleur, matériau et lumière : la pensée chromatique dans l'Égypte ancienne », Technè [En ligne], 40 | 2014, mis en ligne le 24 juillet 2020, consulté le 08 février 2022. URL : http:// journals.openedition.org/techne/3166 ; DOI : https://doi.org/10.4000/techne.3166

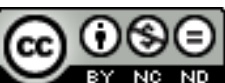

La revue Technè. La science au service de l'histoire de l'art et de la préservation des biens culturels est mise à disposition selon les termes de la Licence Creative Commons Attribution - Pas d'Utilisation

Commerciale - Pas de Modification 4.0 International. 
Christophe Barbotin

\section{Couleur, matériau et lumière : la pensée chromatique dans l'Égypte ancienne}

Colour, material and light: chromatic reasoning in Ancient Egypt

Résumé. Afin d'étudier une civilisation qui attachait autant d'importance à la couleur que l'Égypte pharaonique, la compréhension de l'idée que les Anciens en avaient concue apparaît déterminante. On s'aperçoit ainsi qu'ils prenaient non seulement en compte l'aspect visuel des couleurs, nous dirions leur longueur d'ondes dans le spectre visible, mais aussi la texture et surtout l'éclat des matériaux envisagés, sans distinction particulière entre apparence et nature desdits matériaux. Ils avaient, en outre, établi l'équivalence des couleurs et de la lumière solaire par le biais d'un cheminement intellectuel, non pas scientifique au sens moderne du terme mais hiéroglyphique, ce qui éclaire plusieurs choix iconographiques en décalage par rapport à la nature, et explique l'emploi précis de vernis brillants pour rehausser certains motifs.

Mots-clés. Couleurs, brillance, texture, lumière, simulacres, soleil.

L'Égypte fut une civilisation de la couleur. Les qualités exceptionnelles de son climat nous permettent d'en conserver un aperçu significatif: objets multicolores, bas-reliefs chatoyants sur les murs des temples et des tombes, des palais et parfois des maisons. Elle fut une civilisation de la couleur, mais aussi de la brillance, privilégiant souvent les jeux de contrastes entre le support mat et l'éclat des vernis sur certaines peintures murales ou mobilières, la magnificence des placages métalliques dans la grande architecture, dont les sources nous conservent le souvenir, ou encore les incrustations de «faïence égyptienne», que les Égyptiens nommaient tjehenet, «la resplendissante».

Cette évidence imposée par les données de l'archéologie et de l'histoire de l'art cache toutefois un véritable champ de mines pour l'historien désireux de comprendre de l'intérieur la pensée ancienne. Malentendus et faux-amis se tiennent en embuscade et menacent sans cesse de lui faire subir le sort qu'il redoute le plus: celui du contresens et de l'anachronisme. Heureusement, depuis l'étude fondatrice de Schenkel en $1963^{1}$, notre vigilance ne cesse de s'aiguiser dans ce domaine. Nous savons aujourd'hui combien la perception des couleurs dépend de la langue dans laquelle elles sont conçues, et combien la traduction s'en trouve difficile ${ }^{2}$. Sans reprendre l'ensemble du problème, qui dépasserait trop le cadre de la
Abstract. To study a civilization that attached as much importance to colour as Pharaonic Egypt, understanding the idea that the Ancients had conceived of it seems crucial. One thus realizes that they not only took into account the visual aspect of colours, i.e. their wavelength in the visible spectrum, but also the texture and, above all, the brilliance of the materials envisaged, without making any special distinction between the appearance and the nature of the said materials. They had also established the equivalence between colours and sunlight through a line of thought that was hieroglyphic rather than scientific in the modern sense of the term, which sheds light on several iconographic choices inconsistent with nature and explains the precise use of shiny varnishes to highlight certain motifs.

Keywords. Colours, brilliance, texture, light, simulacra, sunlight.

présente étude, je voudrais attirer l'attention sur quelques aspects spécifiques de la question.

\section{Ces couleurs qui ne sont pas que des couleurs}

La palette fondamentale, dans la pensée égyptienne, se compose du noir (kem), du blanc (hedj), du rouge (decher) et du vert $\left(o u a d j^{3}\right)$. Le vert constitue un cas tout à fait remarquable. Cette notion simple pour nous est rendue en égyptien par le mot ouadj, une racine qui désigne d'abord les plantes fraîches, donc le monde vivant par rapport au rouge du désert (decher) dont il représente la notion antinomique par excellence. Il s'écrit d'ailleurs avec le hiéroglyphe du papyrus qui paraît tout indiqué pour ce service (fig. 1). Et pourtant, le même terme sous la forme ouadj-our, «le grand vert» dans sa traduction courante, définit de vastes étendues d'eau au premier rang desquelles figure la mer. Sauf à être daltonien, il nous est difficile aujourd'hui de concevoir une mer du sud en vert, d'où peut-être l'étrange croisade menée naguère par certains égyptologues contre un élément naturel qui ne leur avait pourtant rien fait ${ }^{4}$ en refusant l'équivalence, même partielle, de ouadj-our et de la mer en dépit de toute évidence. C'est que le champ sémantique du mot doit être réévalué à l'aune des 


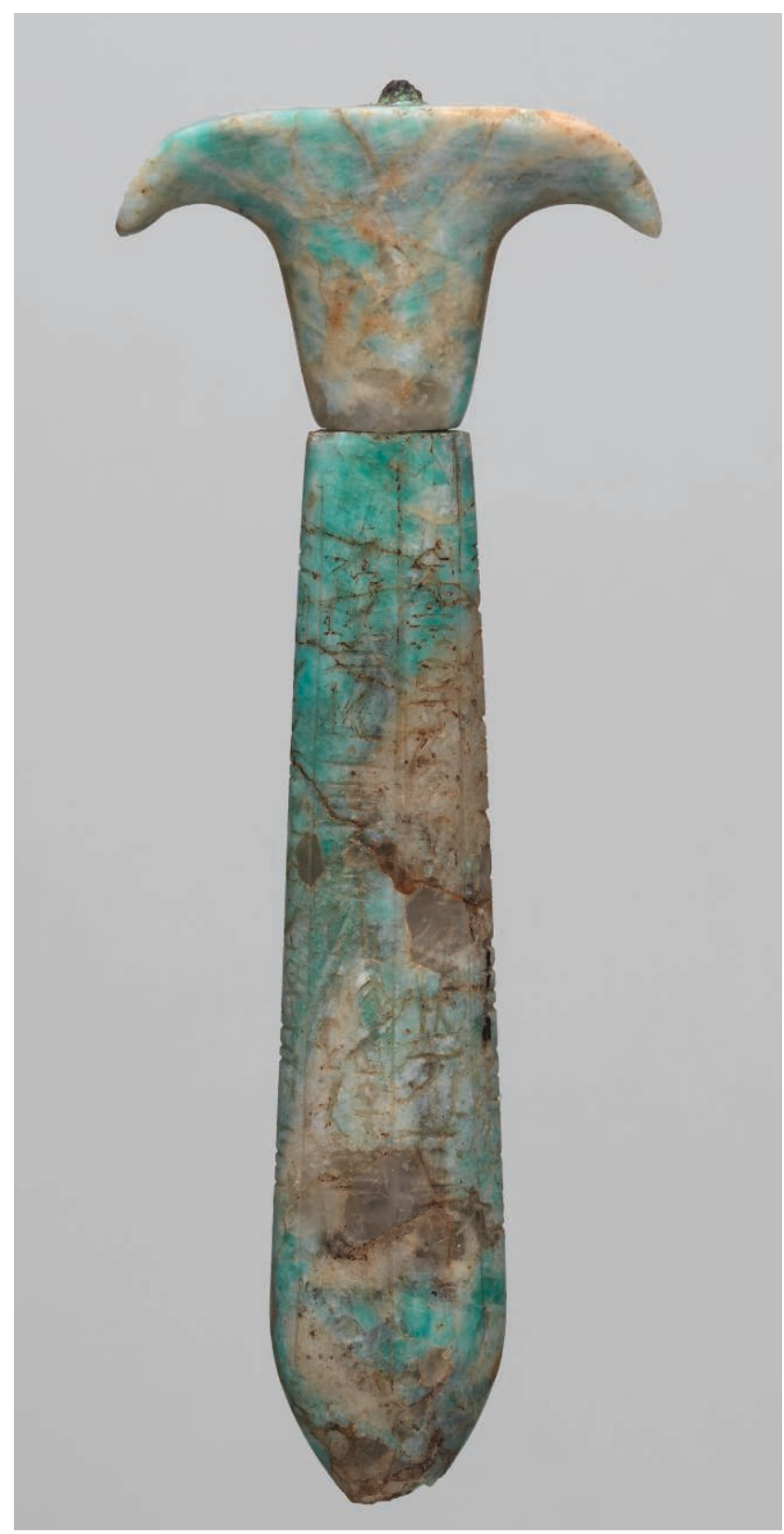

Fig. 1. Amulette ouadj en forme de papyrus, Nouvel Empire, amazonite.

H. 10,2 cm. Musée du Louvre, inv. N 4993. (C) Christian Décamps/ musée du Louvre. modes de perception égyptiens, lesquels ne coïncident pas exactement avec les nôtres. «Le vert une couleur? Oui, mais aussi une lumière $!^{5} »:$ car si le terme ouadj désigne effectivement la couleur verte, il couvre dans le même temps les notions de brillance et de fugacité tant il est vrai que les feuilles de la jeune plante brillent au soleil et que l'eau scintille aux variations du jour ou de la nuit. C'est pour cette raison que la turquoise, qui pour nous est plutôt bleue, est parfois qualifiée de ouadj en égyptien ${ }^{6}$ : d'aspect brillant, sa couleur est fragile et s'altère au soleil ${ }^{7}$. C'est donc le scintillement qui a été retenu pour la qualification de la mer, et non la couleur en elle-même. Plutôt que par «grand vert», ouadj-our devrait en conséquence être traduit par «le grand scintillement» ${ }^{8}$.

La gamme des couleurs égyptiennes ne correspond d'ailleurs pas tout à fait à la nôtre puisque, en dehors de cette palette de base, on relève un absent de marque: le mot «jaune», inconnu ${ }^{9}$ jusqu'au Nouvel Empire, était englobé avant cette date par le rouge (decher ou ines) ou par l'or (noub). Les Égyptiens disposaient en revanche d'un mot spécifique pour le bleu: khesebedj. Ce terme désigne d'abord le lapis-lazuli, pierre bleu sombre importée d'Afghanistan, investie d'une très forte charge symbolique. Le lapis ne se rapporte jamais à la mer, malgré la similitude de sa couleur avec l'azur marin, mais souvent à la nuit. D'abord parce qu'aux yeux des Égyptiens, son caractère foncé le rapprochait du noir ${ }^{10}$. Ensuite parce qu'ils n'ont pas retenu uniquement sa couleur comme caractéristique déterminante, mais aussi la dureté qui rend ce matériau permanent: la perruque bleu-lapis des dieux les désigne comme des êtres éternels, des êtres capables d'agir même dans la grande "suspension» qu'est la nuit ${ }^{11}$. Le mythe de la naissance des rois de la $V^{e}$ dynastie illustre parfaitement la complémentarité de l'or et du lapis. Dans un texte célèbre, on lit en effet comment une simple mortelle met au monde les trois futurs rois destinés à remplacer la lignée de Khéops et à promouvoir le culte du soleil. L'accouchement se fait avec l'assistance d'Isis: «Alors cet enfant glissa sur ses mains comme un enfant d'une coudée. Solides étaient ses os tandis que ses chairs étaient conçues en or et sa coiffe en lapis-lazuli authentique ${ }^{12}$.» Ces rois apparaissent à la naissance avec deux attributs essentiels : la brillance de l'or et la permanence du minéral (fig. 2). Leur chair évoque le soleil, leur coiffe la nuit que celui-ci éclaire. Couleur et matériau se confondent dans la perspective hiéroglyphique qui était celle des Égyptiens, la couleur étant à la fois ce que l'on voit (brillance de l'or et bleu-nuit du lapis) et l'émanation de l'essence ${ }^{13}$ de ce qui est vu (inaltérabilité du soleil et permanence du monde). L'être et le paraître, dans la pensée égyptienne, sont indissociables, ils procèdent de la même nature.

\section{Deux mots pour «couleur»}

La langue égyptienne, outre les termes techniques utilisés pour les pigments ${ }^{14}$, emploie deux mots principaux pour «couleur»: ioun et inem ${ }^{15}$, presque synonymes. Le sens 
Fig. 2. Masque

funéraire de

Toutankhamon, "aux chairs en or et à la coiffe en lapislazuli », fin de la

XVIII ${ }^{\mathrm{e}}$ dynastie, or, lapis-lazuli, cornaline, quartz, obsidienne,

turquoise, verres

colorés. H. $54 \mathrm{~cm}$. Musée du Caire, inv. JE 60672.

(c) BPK, Berlin,

Dist. RMN-Grand

Palais/Margarete Büsing.

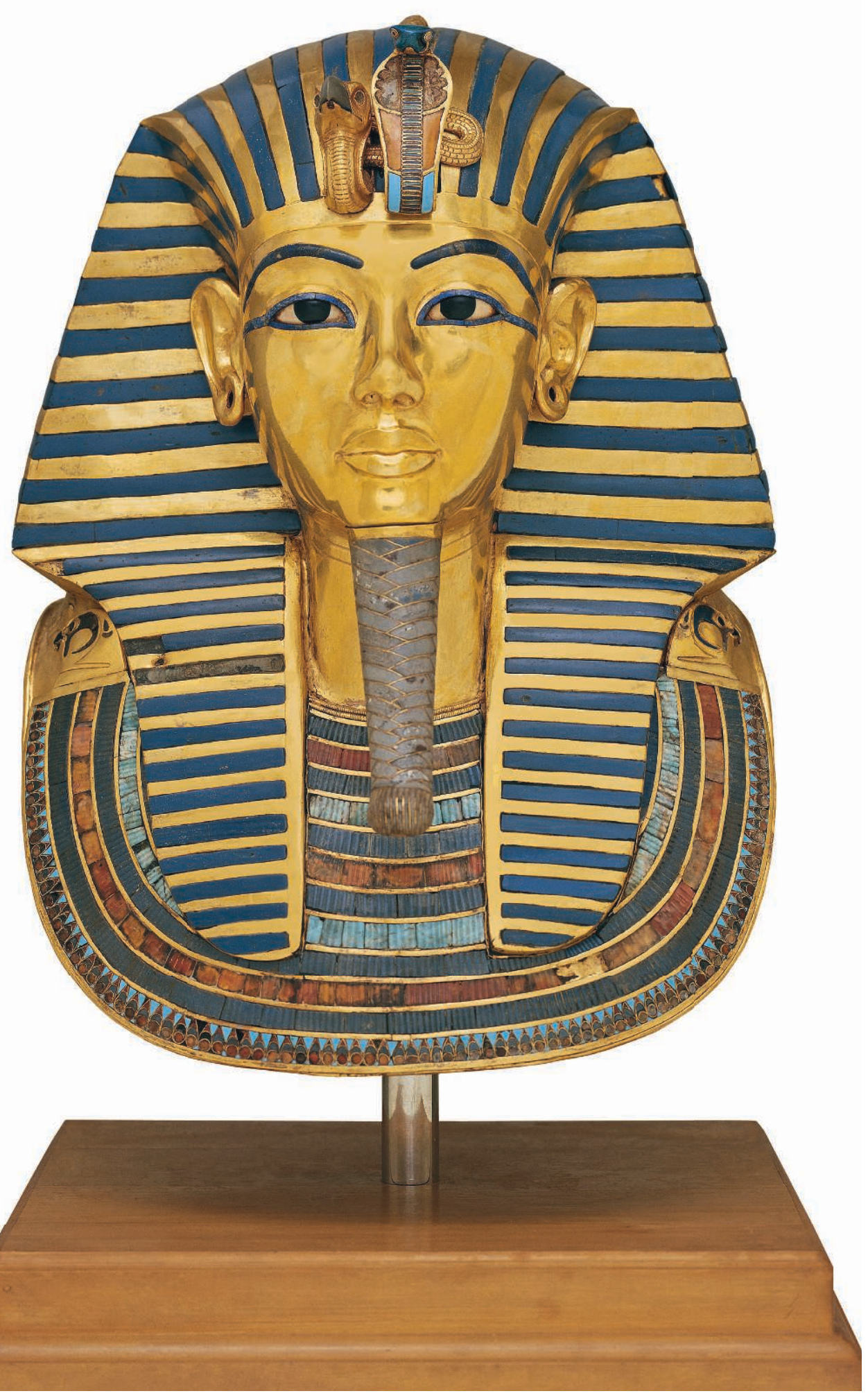




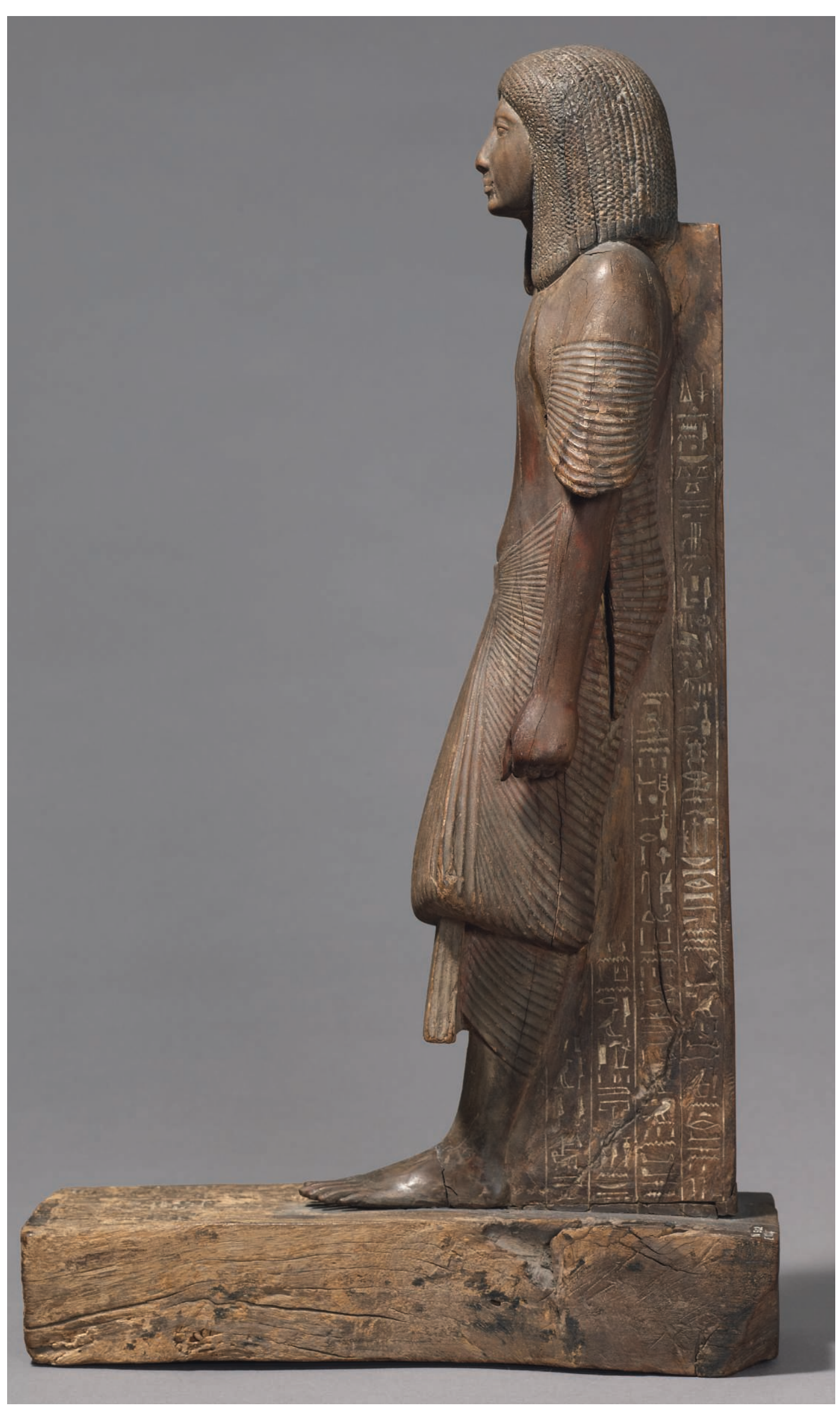

Fig. 3 a. Statue du préposé aux portes Pyiä̈, avec la formule

d'offrande au démiurge sur le côté gauche, fin de la XVIII dynastie, bois. H. $54,3 \mathrm{~cm}$.

Musée du Louvre,

inv. E 124 (N 502).

(C) Christian Décamps/

musée du Louvre. 


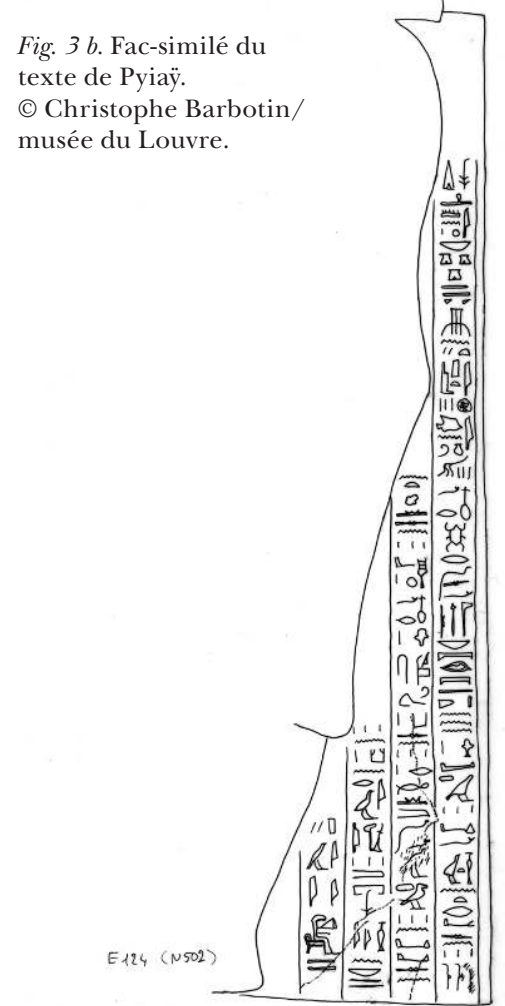

fondamental du premier est «couleur». Par extension, il désigne le «caractère» d'un individu, ce qui implique une identité de nature entre caractère et comportement, entre son individualité et l'apparence qu'il offre au regard ${ }^{16}$. Comme l'a très justement souligné J. Baines, l'existence même d'un terme pour «couleur» dans la langue égyptienne revêt une importance capitale, car elle constitue une part structurante de l'image, donc de la pensée ${ }^{17}$. Il est d'autant plus étonnant qu'il y en ait deux pour une langue aussi pauvre en termes abstraits que l'ancien égyptien. Le sens fondamental du second d'entre eux, inem "peau ", demeure, il est vrai, concret et matériel ${ }^{18}$. Mais à partir de cet aspect visible de l'être humain, inem acquit le sens général de «couleur» à part entière.

\section{Les couleurs comme expression de la lumière}

La lumière constitue un aspect crucial et peu étudié de la pensée chromatique égyptienne ${ }^{19}$. Je voudrais présenter ici un texte méconnu, gravé sur la statue de bois d'un dignitaire de la fin de la XVIII' dynastie conservée au Louvre ${ }^{20}$ (fig. 3a). La première colonne de la formule d'offrandes livre le texte suivant: «Veuille le roi accorder une offrande à Amon-Rê (...), couleur accomplie qui est advenue d'elle-même, le grand dieu seigneur du ciel qui a créé le ciel, la terre et l'eau sur les montagnes (...) » (fig. 3b). Amon-Rê, le seigneur de Karnak, apparaît dans ce texte comme le démiurge à l'origine de l'univers, un dieu-soleil par définition. Cet aspect solaire se concentre dans l'épithète ioun nefer kheper djesef, «couleur accomplie advenue d'elle-même». L'équivalence entre la couleur d'une part, et d'autre part la lumière du soleil à l'aube des temps se trouve ainsi posée sans la moindre ambiguité ${ }^{21}$, elle découle de l'observation de l'aube de chaque jour lorsque, durant quelques minutes, le soleil rouge semble embraser les montagnes du désert. Un hymne proclame en effet que le soleil est «apparu en gloire au point du jour en sorte que les Deux Terres [l'Égypte] sont illuminées de sa couleur $^{22}$ ». Les couleurs constitutives du prisme de la lumière sont une réalité physique que les Égyptiens ne pouvaient certes pas connaître, mais leur pensée chromatique leur en a donné le pressentiment. Ce phénomène suppose d'importantes conséquences quant à notre compréhension de l'iconographie antique. Ainsi, par exemple, le fait que les vautours au plafond des monuments ou les faucons d'Edfou planant au linteau des portes sous forme de disque ailé arborent un plumage multicolore (fig. 4). Ceci n'a rien de naturaliste, puisque de tels oiseaux de proie possèdent dans la réalité un plumage plutôt terne fait de brun, de noir ou de gris. Leur bigarrure prend au contraire le sens d'un hiéroglyphe à part entière signifiant la lumière du ciel dans lequel ils évoluent. Ce hiéroglyphe est d'ailleurs souligné par l'épithète «au plumage multicolore» (sab chout) maintes fois attribuée à ces grands oiseaux célestes, la multiplicité des couleurs étant le seul mode possible d'expression graphique de la lumière solaire. Ce n'est qu'à l'époque amarnienne, au XIV siècle av. J.-C., qu'on a franchi le pas vers la représentation directe de cette dernière par le biais des rayons du soleil. Jusque-là

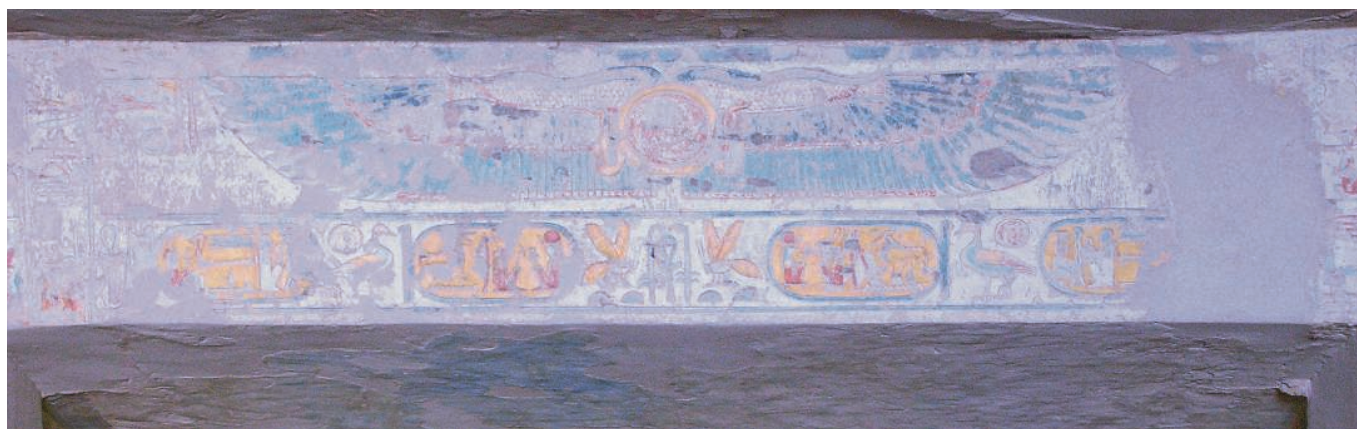

Fig. 4. Le soleil d'Edfou aux ailes de faucon planant dans l'axe de la tombe du pharaon Merenptah, Vallée des Rois, $\mathrm{n}^{\circ} 8$, fin du XIII ${ }^{\mathrm{e}}$ siècle av. J.-C. (C) Pierre Brudieux. 


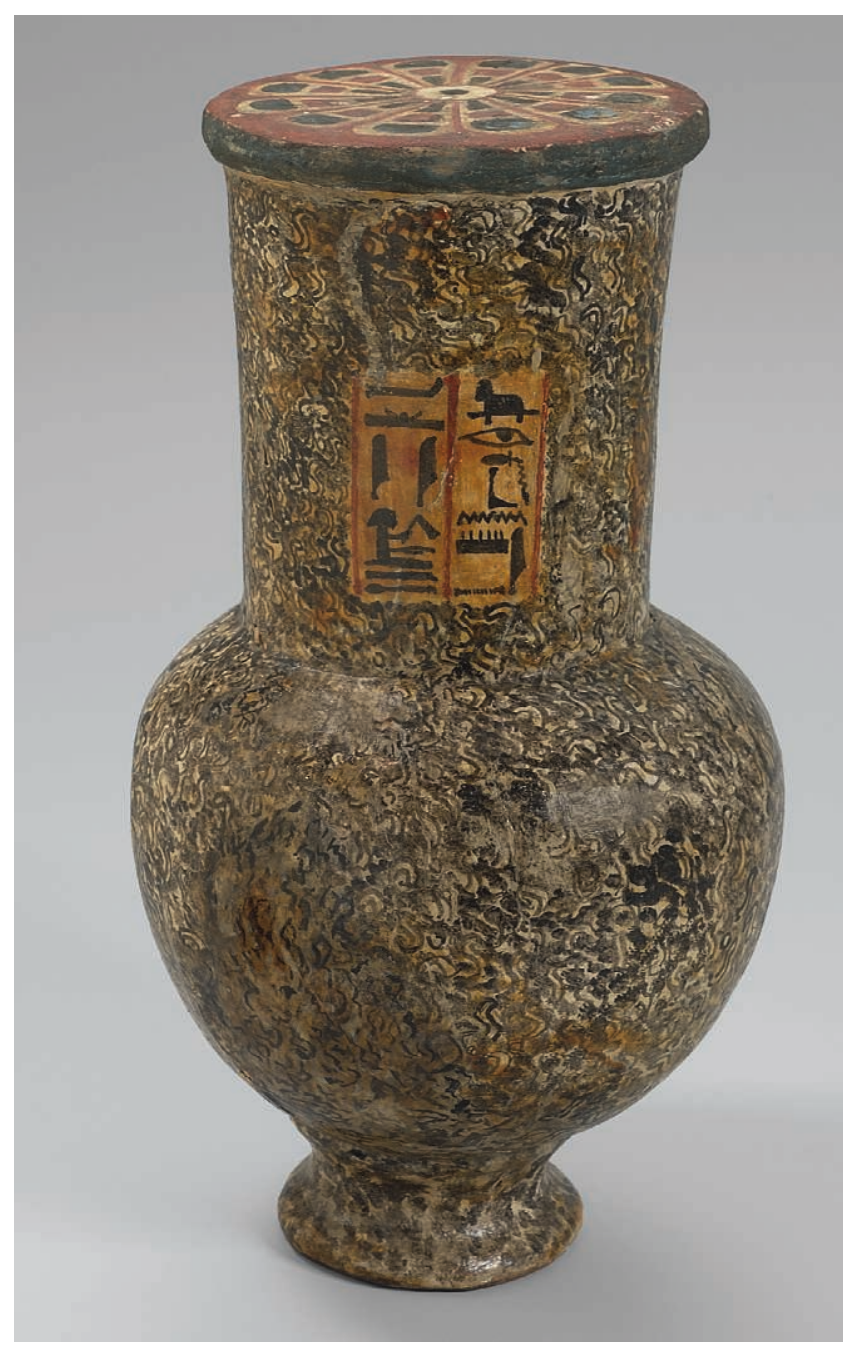

Fig. 5. Simulacre de vase dont la peinture imite l'aspect d'une pierre dure, Nouvel Empire, bois polychrome. H. 27,1 cm. Musée du Louvre, inv. N 932 (AF 209).

(C) Christian Décamps/musée du Louvre.

confinés au seul registre de l'écriture, ils envahissent alors toutes les formes de représentation du monde et chassent des temples et des tombes les images de rapaces aux plumes chamarrées.

Ces notions de base, implicites dans la mise en œuvre de l'art égyptien, n'ont jamais été formulées clairement: il aurait fallu pour cela une pensée philosophique qui n'existait pas dans le monde préhellénique. Mais elles doivent impérativement être prises en compte pour la compréhension des pratiques artistiques égyptiennes.

\section{La lumière rendue par l'emploi des vernis}

Ces notions expliquent notamment l'emploi très sélectif des vernis, que ce soit sur des peintures murales ou sur des objets mobiliers ${ }^{23}$. Le terme égyptien pour vernis est «huile» (merehet), c'est-à-dire un corps transparent plus ou moins fluide. À partir du Nouvel Empire au moins, on sait que cette «huile» donnant les vernis jaunes était essentiellement constituée de résine de pistachier importée de Syrie-Palestine, tandis que les vernis noirs étaient plus divers dans leur composition. La résine de pistachier, incolore à l'état naturel, prend une teinte jaune-orangé lorsqu'elle est chauffée ${ }^{24}$, opération indispensable pour la rendre fluide en vue de son application. Elle donne alors au motif qu'elle recouvre un éclat très soutenu. Or il est établi que cette même résine de pistachier était brûlée dans les cérémonies de culte sous le nom de «ce qui rend divin " (senetjer) ${ }^{25}$, terme générique que nous traduisons habituellement par encens. L'équation des couleurs et de la lumière se trouve donc établie par la brillance du vernis: vernir un objet n'est pas seulement un acte artisanal, c'est plus encore une fonction performative qui confère à l'image une valeur surnaturelle, indéfectiblement attachée au dieu-soleil créateur de l'univers. Vernir un sujet revient à le «rendre divin» (senetjer), c'est un acte de même nature que brûler cette résine dans un cadre rituel, lorsque la fumée odorante matérialise l'espace-lumière au sein duquel la divinité ou le défunt pourra exercer son action bénéfique en faveur des hommes ${ }^{26}$. Voilà pourquoi certaines scènes de tombes thébaines érigent le vernissage d'un cercueil au rang d'acte rituel supervisé par un prêtre ${ }^{27}$ !

\section{Couleurs et simulacre}

On considèrera comme une autre conséquence de la pensée chromatique égyptienne, de cette interdépendance de l'être et du paraître qui lui est propre, l'usage récurrent que les Égyptiens ont toujours fait du simulacre. Simulacres d'architecture, de sculpture ou de peinture: fausses chapelles dans le complexe funéraire du roi Djéser à Saqqara afin de perpétuer l'efficience des cérémonies de jubilé, fausses portes dans les tombes qui doivent permettre au $b a$ du mort, son principe mobile, de passer d'un monde à l'autre, emploi de couleurs parfois très complexes pour simuler un matériau qui n'est pas celui de l'objet. Les Égyptiens étaient passés maîtres dans l'art de rendre du faux bois, du faux granite (fig. 5) ou du faux métal à l'aide de peinture. Nous appelons ces couleurs de substitution «fausses» par commodité de langage, alors qu'aux yeux des Égyptiens, elles remplissaient une fonction identique à l'original, sans intention de tromperie. Le vocabulaire se fait parfois l'écho de ces matériaux de remplacement: les Annales du pharaon Thoutmosis III distinguent le «lapis-lazuli authentique " (khesebedj maâ), c'est-à-dire le minéral en tant que tel, du «lapis-lazuli qui a été fait» (khesebedj $i r y t)$, où l'on reconnait la faïence égyptienne, et du «lapis-lazuli fondu » (khesebedj oudehou), qui se rapporte à la pâte de verre. La turquoise, elle aussi, pouvait être «authentique» ou «fondue». C'est ce mode de pensée qui permettait de remplacer les matériaux précieux par une couleur ordinaire avec une fonctionnalité identique. Après le sac des tombes de la Vallée des Rois, au XI ${ }^{\mathrm{e}}$ siècle av. J.-C., les prêtres d'Amon replacèrent ainsi les momies des pharaons dans des cercueils de bois rehaussés de peinture jaune pour remplacer l'or d'origine. 
De même, un cercueil du début de la XXII ${ }^{\mathrm{e}}$ dynastie, au British Museum ${ }^{28}$, reçut un fond gris imitant l'argent, fond sur lequel les figures se détachent avec éclat grâce au vernis jaune brillant dont il a été question plus haut: effet saisissant qui évoque immédiatement un ouvrage d'orfèvrerie.

On voit combien la notion de couleur pour les anciens Égyptiens ne saurait se réduire à ce que nous appelons aujourd'hui une longueur d'ondes dans le spectre visible: elle inclut tout autant la texture, la durabilité et surtout la lumière que des assemblages multicolores permettaient d'exprimer en tant que telle. Tels sont donc quelques-uns des principes sous-jacents qui guidèrent dans leur tâche artistes et restaurateurs de l'ancienne Égypte.
Notes

1. Schenkel, 1963, p. 131-147.

2. Pour une excellente synthèse sur le mode de pensée chromatique des anciens Égyptiens, voir Mathieu, 2009. On ajoutera utilement à sa bibliographie (op. cit. p. 50-52) l'étude de Spalinger (Spalinger, 2008, p. 239-245) et celle de Warburton (Warburton, 2008)

3. Schenkel, 1963, p. 143-144, et Pinch, 2001, p. 182.

4. Le bréviaire de cette épopée étant Vandersleyen, 1999 (avec une couverture verte...).

5. Gautier, 1997, p. 14

6. Quirke, 2001, p. 188-189.

7. Texte de Horourrê au Sinaï (cité par Quirke, 2001, p. 187). A contrario, certaines sources parlent de "turquoise neuve», conséquence de la fragilité de la couleur de ce minéral.

8. On trouve un cas similaire avec la «mer vineuse» d'Homère (épi oïnopa ponton, Iliade 1, 350 et Odyssée 1, 183), une comparaison qui ne saurait tirer son origine d'une couleur que la mer ne prend jamais.

9. Schenkel, 1963, p. 142.

10. Mathieu, 2009, p. 26-27 et 47 .

11. Le mot «nuit» est construit sur la racine gereh qui signifie «cesser».

12. Papyrus Westcar (Berlin 3033), 10,10-10,11. Pour le lapis-lazuli «authentique», voir infra.

13. Il y a un jeu sur le mot nekhebet, rendu ici par «conçu», qui désigne ordinairement l'ensemble des noms du roi, c'est-à-dire sa nature profonde, son essence. Par le biais du nom, l'or et le lapis concourent à la définition de cette nature.

14. Mathieu, 2009, p. 25, n. 2.

15. Mathieu, 2009, p. 25.

16. De même, le mot qed désigne à la fois «la forme» que l'on voit, et le «caractère» qui identifie un individu, être et paraître étant de nature identique.

17. Baines, 2007, p. 259-260.

18. C'est ce terme qui est employé par
Horourrê pour qualifier l'aspect de la turquoise (voir supra n. 7).

19. Gautier, 1997, p. 14.

20. Statue du portier Pyiaÿ, inv. E 124

(N 502), exposée dans la salle 26 du département des Antiquités égyptiennes.

21. De même, la couleur ioun est associée à la forme (qi) du démiurge dans l'hymne à Amon du papyrus Leiden I 350 , recto II, ligne 26: «(Amon) qui s'est façonné lui-même, couleur parfaite (ioun nefer) qui s'est manifestée en aspect inaccessible » (Zandee, 1947, pl. II, 1. 26), ainsi que dans un autre hymne à Amon sur ostracon (Černý, Gardiner, 1957, pl. CVI, 1, recto 4). 22. Dorn, 2011, ostracon 746 b, p. 458 et pl. 654-656.

23. Sur les vernis égyptiens au Nouvel Empire, voir Serpico et White, 2001.

24. Serpico et White, 2001, p. 35. 25. Serpico et White, 2001, p. 36. 26. Les Égyptiens ne concevaient l'air qu'en mouvement, «le souffle». Ce que nous appelons atmosphère était pour eux du «vide» (chou) que la lumière du soleil venait animer chaque matin. Il est significatif qu'aucun acte rituel ne se déroule dans le temple pendant la nuit, la fumée de l'encens est inséparable de la lumière du jour.

27. Serpico et White, 2001, p. 37. Dans la tombe de Neferhotep, c'est la préparation de la résine qui est sacralisée, tandis que dans celle de Tchay, c'est son application sur la momie.

28. Inv. EA 6660 : Serpico et White, 2001, p. 35.

\section{Bibliographie}

Baines J., 2007, Visual and Written Culture in Ancient Egypt, Oxford University Press, Oxford.

Černý J, Gardiner A. H., 1957, Hieratic Ostraca, The Griffith Institute, Oxford.

Dorn A., 2011, Arbeiterhütten im Tal der Könige, Schwabe AG Verlag, Bâle.
Gautier P. A., 1997, «Le rouge et le vert: sémiologie de la couleur en Égypte ancienne", Archéo-Nil, 7, p. 9-15 (position de thèse).

Mathieu B., 2009, «Les couleurs dans les Textes des Pyramides: approche des systèmes chromatiques (enquêtes dans les Textes des Pyramides, 2) ", Égypte Nilotique et Méditerranéenne, 2, p. 25-52.

Pinch G., 2001, "Red things: the symbolism of colour in magic", dans Davies W. V. (éd.), Colour and Painting in Ancient Egypt, The Trustees of the British Museum, Londres, p. 182-185.

Quirke St., 2001, "Colour Vocabularies in Ancient Egypt”, dans Davies W. V. (éd.), Colour and Painting in Ancient Egypt, The Trustees of the British Museum, Londres, p. 186-192.

Schenkel W., 1963, «Die Farben in ägyptischer Kunst und Sprache», Zeitschrift für ägyptische Sprache und Altertumskunde, 88, p. 131-147.

Serpico M., White R., 2001, "The use and identification of varnish on New Kingdom funerary equipment", dans Davies W. V. (éd.), Colour and Painting in Ancient Egypt, The Trustees of the British Musuem, Londres, p. 33-42.

Spalinger A., 2008, "Colors and Directions", dans Waitkus W. (éd.), Diener des Horus. Festschrift für Dieter Kurth zum 65. Geburtstag, PeWe, Hambourg, p. 239245.

Vandersleyen Cl., 1999, Ouadj-our. Un autre aspect de la vallée du Nil, Connaissance de l'Égypte ancienne, 7, Bruxelles.

Warburton D., 2008, “The Theoretical Implications of Ancient Egyptian Colour Vocabulary for Anthropological and Cognitive Theory", Lingua Aegyptia. Journal of Egyptian Language Studies, 16, p. 213-259.

Zandee J., 1947, De hymnen aan Amon van Papyrus Leiden I 350, Rijksmuseum van Oudheden te Leiden, Leyde. 